\title{
BOND-GRAPHS AND GENETIC ALGORITHMS FOR DESIGN AND OPTIMIZATION OF ACTIVE DYNAMIC SYSTEMS
}

\author{
Amara Elhoucine ${ }^{1}$, Faiçal Miled ${ }^{2}$, Kamel BenOthman ${ }^{3}$ \\ ${ }^{1}$ PHD Student, ${ }^{2}$ Assistant Professor, ${ }^{3}$ Master of conferences, LARATSI, ENIM (National School of engineering of \\ Monastir), Tunisia \\ Houcine.Amara@enim.rnu.tn,f.miled@uha.fr, Kamelbenothman@yahoo.fr
}

\begin{abstract}
The aim of this work is to propose a methodology of sizing in a frame of the conception of mechatronic systems in particular and active dynamic ones, in general. This methodology will support conceptual design step [1]. We propose a collaborative design approach based on Bond Graph tool and Genetic algorithms. Mechatronic systems have a passive part and active part. In our approach we establish a hard interaction between passive and active parts. This interaction will be materialized in taking into account of control criteria in the evolution (synthesis) phase of passive part. This initiative treats functional aspects, both structural and behavioral; while respecting interactions between passive and active parts.

In our approach we treat functional, structural and behavioral aspects in order to validate a post-project solution. Optimization needs Behavioral models which are systematically deducted from bond-graph structural models. Thus, retained bond graph elements which constitute passive part will be obtained, done by optimization Genetic Algorithms procedure. In this procedure Gramians of controllability and of observability represent the fitness function. The proposed method will be applied to an automatic transmission of a scooter and validated by a dynamic simulation.
\end{abstract}

Keywords: Modeling, dynamic system, Bond graph, Gramians of controllability, Genetic algorithms

\section{INTRODUCTION}

The optimization of mechatronic systems [2] makes part of the design research [3, 4]. Actually, design methodology use optimization in routine design when we modify some values of structural components and validate by simulations $[5,6]$. In our approach we use optimization procedure in a creative design which starts form initial specification.

This procedure will be integrated in a mechatronic design methodology [7] supported by a functional, structural and behavioral steps. Functional step transform initial specification in to functional specification. In the structural step we synthesis passive part represented by bond graph. In the behavioral step we use our optimization procedure to validate our solutions.

The steps of design and dimensional of dynamic systems assets are based on criteria of controller Such as controllability, observability and inversibility. In fact, the controllability we can achieve under optimal conditions of cost and performance control system. The inversibility is essential for the implementation of a number of control laws, including the input-output decoupling and disturbance rejection [8] and to clarify the necessary design equations.
In this article we propose a synthesis sizing method based on dynamic and energetic criteria [9],by exploiting the optimization of Gramians $[9,10,11]$ for the formulation of an optimization problem and its resolution by the method which reveals out from artificial intelligence; in this case genetic algorithms.

The first part of this article deals with an approach which leads to a functional model and then structural in the direction of bond-graph (casual model). This model is synthesized by imposing to the recent structural criteria of controller.

The second part of this article presents a behavioral and quantitative synthesis in order to size elements bond-graph of the passive part. In this frame we base ourselves on both dynamic and energetic criteria while respecting the specifications. That's why we are in front of a problem of optimization of Gramians of controllability and observability, which presents the objectif function, the optimization of which is supported by the genetic algorithms.

The third part consists in making a dynamic simulation to validate our method of sizing of which we determine the laws of controllability which require the operation of the inverse bond-graph which is based on the concept of bi-causality [12]. 


\section{FUNCTIONAL AND STRUCTURAL STUDY}

Functional model is obtained after transforming technical functions into bond-graph sub-models [7]. Interconnection of sub-models from an a-causal bond graph model which considered as a functional one [13]

The Bond Graph tool is a unified graphic language [13] for all the domains of engineering sciences and confirmed as an approach structured in the modeling and in the simulation of the multidisciplinary systems. We chose as application an automatic transmission of a scooter. Its global function is to transform engine power into back wheel motion [7].

We propose two functional models. An initial model validated in a precedent study [7] and a candidate model constituted by an R, C and MSe elements. After structural study, we will apply our optimization procedure to size $\mathrm{R}$ and $\mathrm{C}$ elements.

We chose as application an automatic transmission of a scooter of a scooter. Its functional model is established by three sub-functional models (Fig.1) (engine, transmission and load) [7].

We can classify the functional models in two categories:

- Model without element E

- Model with element E

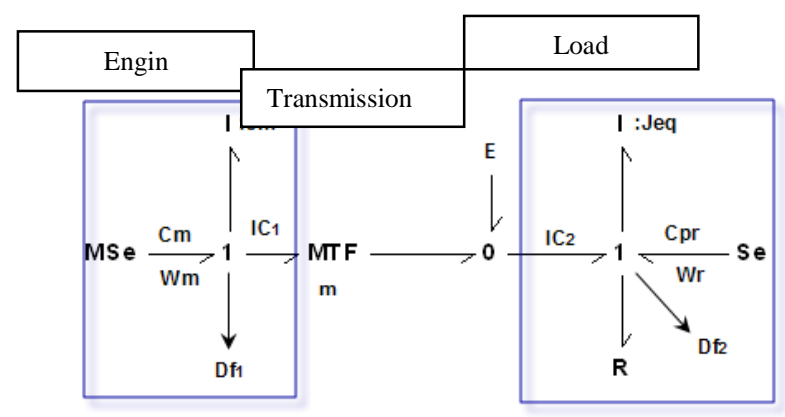

Fig. 1: Model candidate of automatic transmission of scooter [7]

$\mathrm{J}_{\mathrm{eq}}$ : represents the equivalent rotation inertia applied to the back wheel. It takes into account the mass of the scooter, the user and the rotational inertia of the wheel;

$\mathrm{J}_{\mathrm{m}}$ : moment of inertia of the apparent engine relative to its output shaft $\left(\mathrm{Kg} / \mathrm{m}^{2}\right)$;

$\mathrm{I}_{\mathrm{C} 1}$ : link connecting the engine with the transmission;

lc2: the connexion link between the transmission and back wheel.

$\mathrm{R}=\mathrm{R}\left(\omega_{\mathrm{r}}\right)=-0 \cdot 5 \cdot C x \cdot \rho \cdot S \cdot \mathrm{R}_{1}{ }^{3} \cdot \omega_{\mathrm{r}}$; element bond graph that defines the action due to penetration of the whole scooter, driver and passenger in the air;

$\mathrm{C}_{\mathrm{pr}}=-\mathrm{m}_{\mathrm{t}} \mathrm{g} \sin \theta \mathrm{R}_{1}$; torque generated by the road profile;

$\mathrm{m}_{\mathrm{t}}$ : total mass of the scooter;

$\theta$ : Slope value; $\rho$ : Air density;

$\mathrm{R}_{1}$ : ray of the back wheel;

$\mathrm{C}_{\mathrm{x}}$ : coefficient of resistance;

$\mathrm{S}$ : frontal surface of contact with the air;

$\omega_{\mathrm{r}}$ : angular velocity of back wheel.

The bond graph causal model is considered (D.Tanguy et al 2000) as a structural model. We synthesise the structural model (Rahmani, 1993) by taking into account control criteria (controllability, observability and invertibility).

In what follows we are going to work on a model with an element $\mathrm{E}$ which will be retained as a model candidate for the sizing. The identification of the nature of the element $\mathrm{E}$ is made during the structural analysis. Indeed, the element $\mathrm{E}$ has to contribute to the establishment of the structural properties of control which the designers impose on the passive part.

The structural models are bond-graph causal models. The causality allows creating explicitly the relations of cause and effect, and the structure of calculation of the characteristic equations associated to the models. For this study, we have to retain a model which has to verify the criteria of controllability of observability and structural inversibility. The model of our conception is the one of the transmission, which has to contribute to the check of these criteria. At a first level, to have the compulsory structural properties, the element $E$ has to be a modulated source of effort. Then, we make an $\mathrm{U}_{2}$ control [7] (Fig.2) associated to this element E.

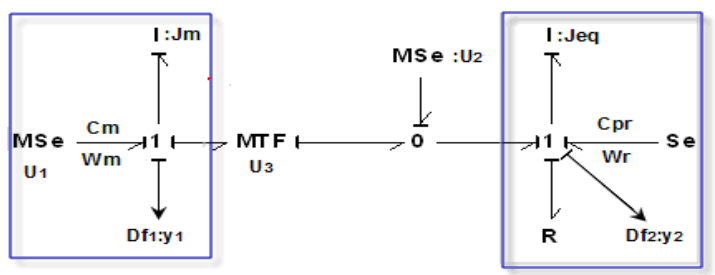

Fig.2: Model candidate [7].

To improve the performances and possibly to limit the use of power among the source of controller $\mathrm{U}_{2}$ we propose at a second level the following model (Fig.3)

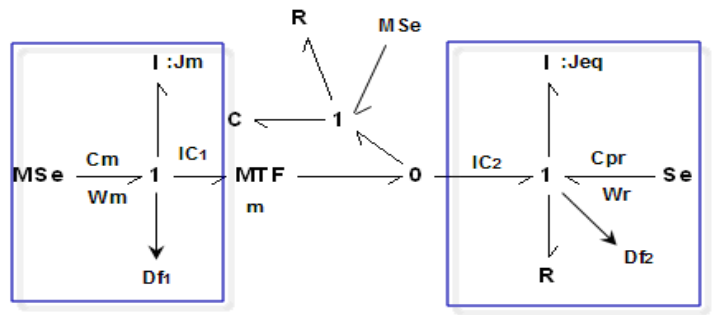

Fig. 3: Proposed Model 


\subsection{Consideration of Controllability}

To put the model in model BGD, it is necessary to make duality sources. Thus the criterion of controllability is verified with dualization of $U_{1}$ which we consider as being a source of controller (Fig.4).

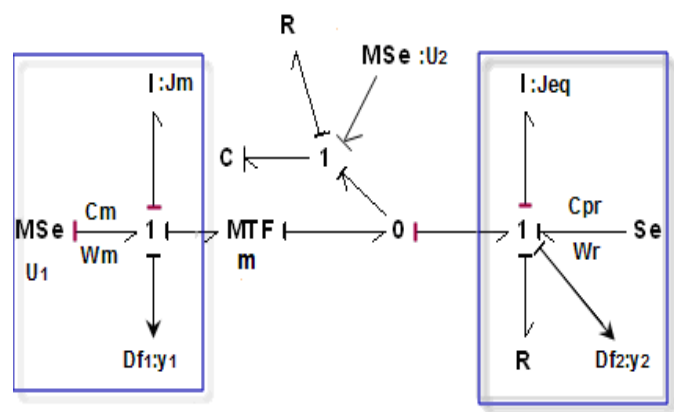

Fig. 4: Model BGD (derived bond-graph)

\subsection{Consideration of the Inversibility}

According to the criteria of inversibility of A. Rahmani [12], we should have the same number of inputs and outputs. The proposed model is established by two outputs y1 and y2 (respectively the rotation speed of the engine and the wheel), two inputs which are U1 and U2. By applying the procedures of consideration of the inversibility, we deduct the shortest causal paths connecting the inputs and the outputs (Fig 5).

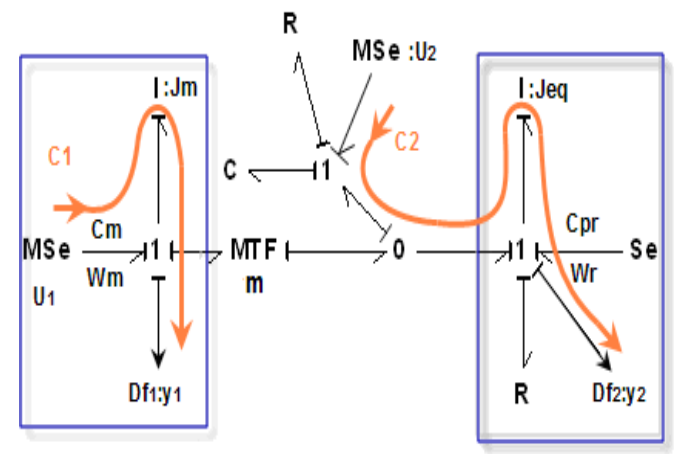

Fig. 5: The causal roads connecting the input with output

\subsection{Consideration of the Observability}

The first step of the procedure of consideration of the observability is the stake of the model bond-graph in BGI. At first, it is necessary to verify the existence of a causal path between every sensor and a dynamic element (I or C) and making all the dynamic elements in complete causality during BGI (Fig.6).

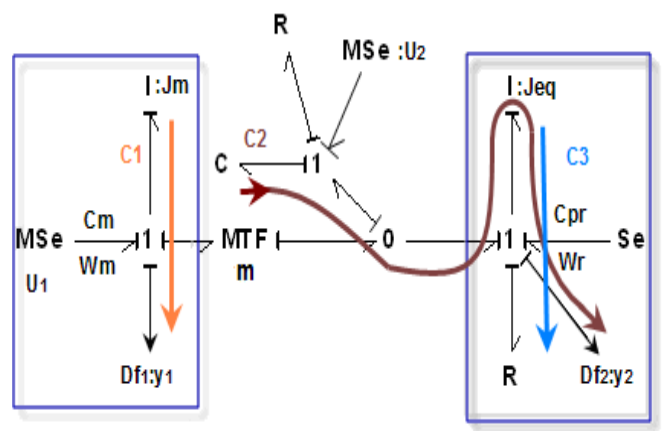

Fig.6: The causal roads every element C or I and the sensors.

The second step consists in putting the model in BGD with the dualization of the sensors.

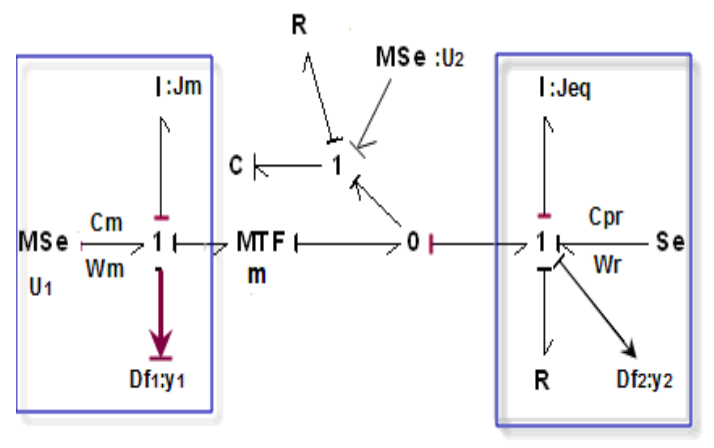

Fig.7: Model BGD (derived bond-graph)

To assure the criterion of observability, it is necessary to dualise the sensor $D^{*} f_{1}$, the relatives to y1.

After verifying structurally our model, we pass to the following step which consists in making a study behavioral of our model with the aim of sizing the passive elements of the model candidate.

\section{BEHAVIORAL STUDY}

In our days the sizing of the systems is made by a set of simulation either by modifying the structure by programs intelligent as the genetic programming $[6,14]$, or by modifying the parameters of the structures while limiting itself to a compromise between the performances wished and the power of actuators [12] These practices engender a conception under optimal as far as they do not take into account the interactions between passive and active elements.

A current method of sizing $[6,15]$ of the systems is made by a set of simulation either by modifying the structure by programs intelligent as the genetic programming. Other used 
methods are based on opposite bond-graph [12] which consists in imposing the input to find output.

Nevertheless these methods are limited juts to the sizing of actuators. Furthermore, several criteria are used such as the energy criteria [9].

We propose an approach for dimensioning passive part based on genetic algorithms. We also use control an energetic criteria such as Gramians of controllability and observability. These criteria allow to minimize input power and to maximize output power. For our case, we propose an approach of dimensioning of the passive elements which is based on the genetic algorithms in order to optimize Gramians of controllability and observability via the energy of controller.

\subsection{Proposed Method}

Indeed, in the case of the dynamic systems, the minimal energy to be supplied to the system to reach a given state is inversely proportional in Gramian of controllability and that the energy of output generated by a given initial state is proportional in Gramian of observability [13].

There are several tools of optimization among which determinists (method of gradient) [16] and the other heuristics (Algorithms genetics) [15,17].

With the aim of optimizing these Gramians we are going to use the algorithm genetic as a tool of optimization.

The proposed method of sizing amounts in the organization chart following (Fig.8):

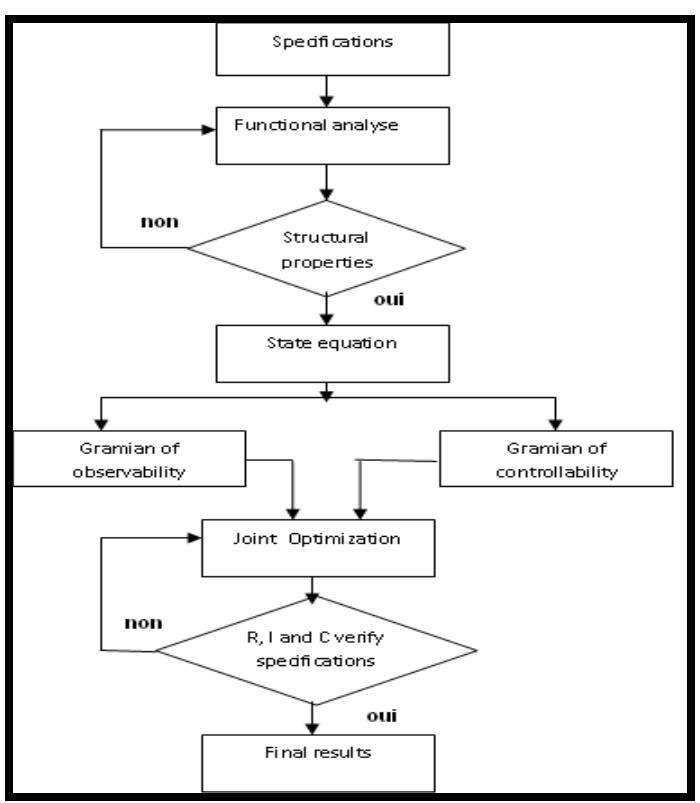

Fig.8: The proposed dimensioning procedure
To size elements $\mathrm{R}$ and $\mathrm{C}$ held in our model of the automatic transmission of scooter we are going to follow the steps of the figure:

- Synthesis of the equations of state from the structural model.

- Calculation of Gramians of controllability and observability from the model of state.

- Joint Optimization of the Gramians of observability and of controllability by genetic algorithms.

\subsection{Algorithms Genetic}

The genetic algorithms are tools of optimization based on a mechanism of disturbance, a criterion of evaluation and a criterion of break [17]. These genetic algorithms are based on techniques derived of the genetics and the natural evolution of Darwin: crossings, transformations, selection, etc.

The functioning of the genetic algorithm can be presented on the following organization chart (Fig.9)

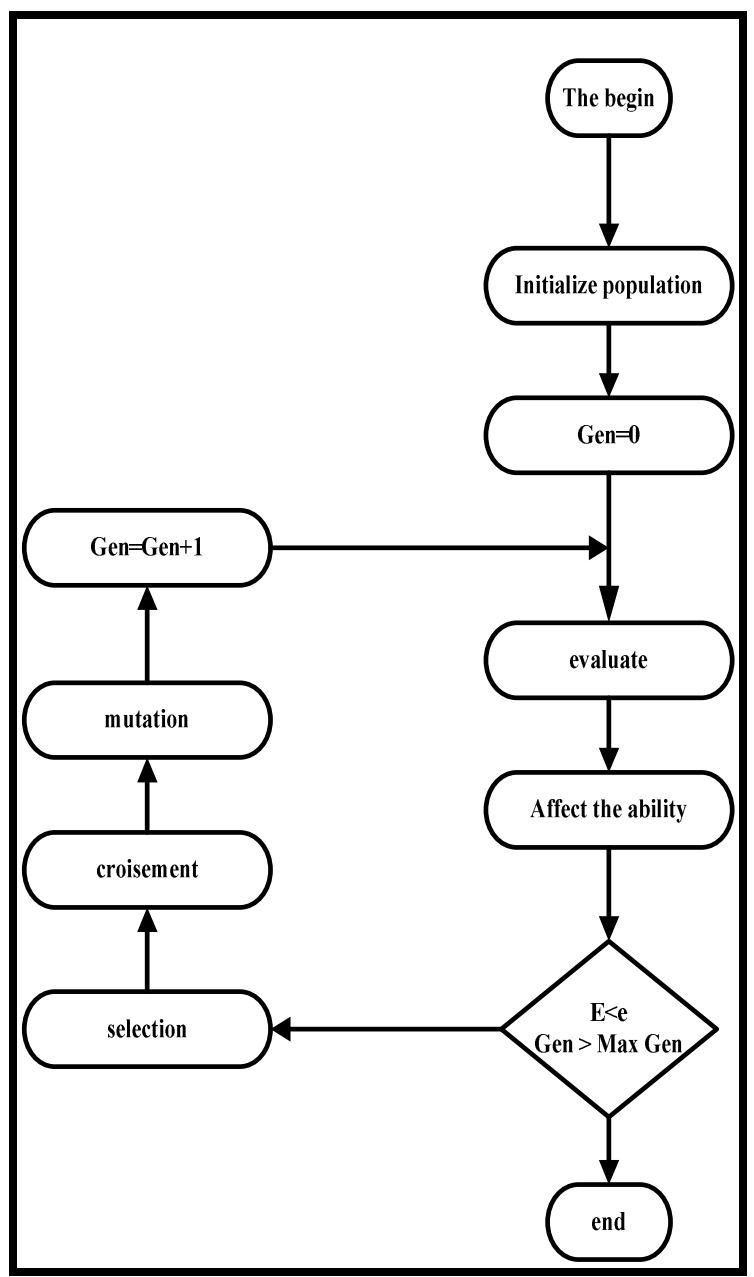

Fig. 9: Organization chart of functioning of algorithms 
The problem is defined by:

Minimize $f$ (I) under the constraints:

$\left\{\begin{array}{l}h_{j}(I) \leq 0 \\ x_{i} \in I \\ x_{i \min } \leq x_{i} \leq x_{i \max }\end{array}\right.$

With:

f: objectif function.

$h_{j}$ : The constraints applied to the system.

I: The vector of conception of the independent variables.

$x_{i}$ : The variables of the individual in the terminology of the genetic algorithms.

$x_{i \text { min }} x_{i \max }$ : borders lower and upper of the variables of the individual.

The model bond-graph of the scooter admits the vector of the variables of conception Independents $\mathrm{I}=[\mathrm{R}, \mathrm{C}]$.

After, we are going to apply our approach to the example of automatic transmission of scooter.

\subsection{Application}

In this application, we suggest determining the parameters $\mathrm{R}$ and $\mathrm{C}$ of the model bond-graph of the automatic transmission of scooter (Fi.10):

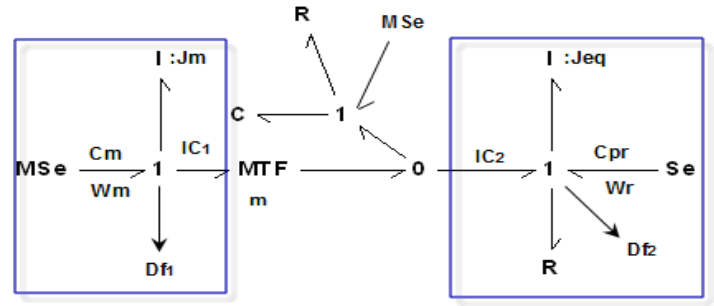

Fig.10: Model Bond-graph of automatic transmission of scooter

\section{Model of State}

$$
\left\{\begin{array}{l}
\dot{x}=A x+B u \\
y=c x
\end{array}\right.
$$

The model of state is obtained by substituting the algebrodifferential equations in the equations of the elementary laws. Matrix $\mathrm{A}$ and $\mathrm{B}$ and $\mathrm{C}$ are given by the following relations:

$$
A=\left(\begin{array}{ccc}
\frac{m^{2} * R_{1}}{J_{m}} & \frac{-m^{2} * R_{1}}{J_{e q}} & \frac{m}{c} \\
\frac{-R_{1}}{J_{m}} & \frac{R_{1}}{J_{e q} * m}-\frac{R}{J_{e q}} & \frac{-1}{c} \\
\frac{m}{J_{m}} & -\frac{1}{J_{e q}} & 0
\end{array}\right) ; \quad B=\left(\begin{array}{cc}
1 & -m \\
0 & \frac{1}{m} \\
0 & 0
\end{array}\right) ;
$$$$
C=\left(\begin{array}{ccc}
\frac{1}{J_{m}} & 0 & 0 \\
0 & \frac{1}{J_{e q}} & 0
\end{array}\right)
$$

This equation of state is the intermediary between the structural analysis and the behavioral analysis.

\section{Result of Optimization}

The interval of existence of the parameters used by the genetic algorithms is given by the following table.

Table .1. Intervals of existence of parameters.

\begin{tabular}{|l|l|l|}
\hline Intervalle & $\mathrm{R}$ & $\mathrm{C}$ \\
\hline $\mathrm{Xmin}$ & 0.1 & 0 \\
\hline $\mathrm{Xmax}$ & 0.5 & 1 \\
\hline
\end{tabular}

The optimal solution, obtained by algorithm genetics (AGs) during the minimization of the trace of Gramian of observability and the maximization of the trace of Gramian of controllability, is presented in the following table.

Table 2: the optimal solution for elements(R and C)

\begin{tabular}{|l|l|l|}
\hline valeurs & R & C \\
\hline$X$ & 0.4227 & 0.3132 \\
\hline
\end{tabular}

After sizing elements bond- graphs ( $\mathrm{R}$ and $\mathrm{C}$ ) the following step consists in validating of our methods.

\section{SIMULATION AND VALIDATION}

In order to validate our method of sizing based on the optimization by genetic algorithm we are going to make a comparative study enter both models:

> Model with elements $\mathrm{R}$ and $\mathrm{C}$ (proposed model: $\mathrm{V}_{1}$ ). Model without elements $\mathrm{R}$ and $\mathrm{C}$ (initial model: $\mathrm{V}_{0}$ ).

So we are going to determine the laws of the controls for every model to clarify the powers put in sets everything. In this executive we run the inverse bond-graph for the calculation of the laws of controller. 


\subsection{Simulation of the Initial Model (without elements $R$ and $\mathbf{C )}$}

To simulate this model we are going to determine the U1 controls (power to be applied of scooter) and U2 (torque to be applied to the back wheel of scooter).

The determination of the law of control requires the operation of the inverse bond-graph which based on the concept of bicausality. Thus the inverse bond-graph of the model without $\mathrm{R}$ and $\mathrm{C}$ is given by the following figure (Fig.11).

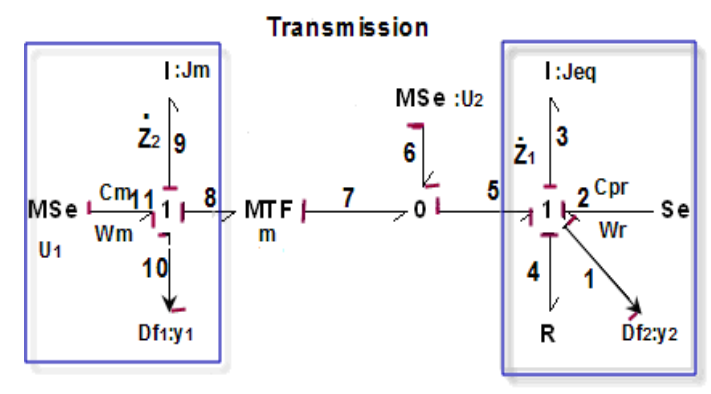

Fig.11: The inverse Bond-graph of initial model V0 (without $\mathrm{R}$ and $\mathrm{C}$ )

By substituting the algebro-differential equations in the equations of the constraints, obtained from the model bondgraph inverse, the controllers $\mathrm{U} 1$ and $\mathrm{U} 2$ are given by the following equation ones.

$$
\left\{\begin{array}{l}
U_{1}=J_{m} p y_{1}+m J_{e q} p y_{2}-m C_{p r}+m R y_{2} \\
U_{2}=J_{e q} p y_{2}-C_{p r}+R y_{2}
\end{array}\right.
$$

By imposing beforehand y1 (speed of scooter in $\mathrm{Km} / \mathrm{h}$ ) and $\mathrm{y} 2$ (rotation speed of the engine in $\mathrm{rad} / \mathrm{s}$ ) two dynamics of the first one order Fig.12 and Fig.13:

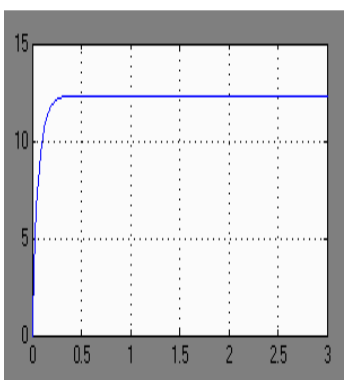

Fig.12.Output y1(The speed of the scooter $(\mathrm{Km} / \mathrm{h})$

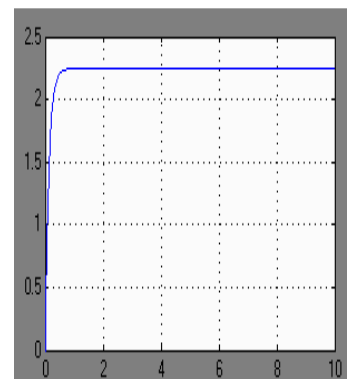

Fig.13. Brought out y2 (the rotation speed of engine $(\mathrm{rad} / \mathrm{s})$

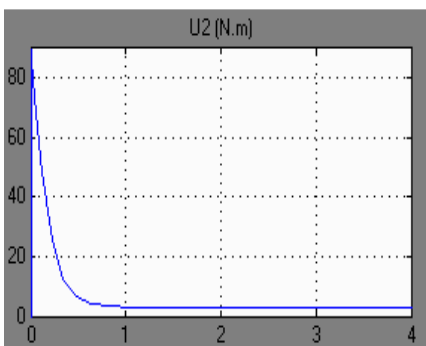

Fig. 14. The U2 controller (N.m)

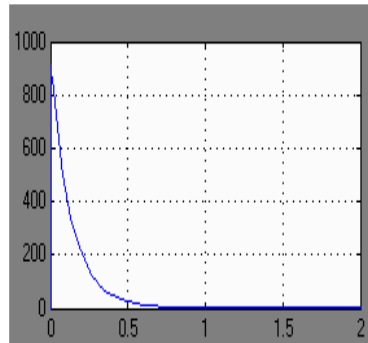

Fig.15. The U1 controller (W).
$\mathrm{U} 2$ is the control which represents the torque to be applied to the back wheel of the scooter.

$\mathrm{U} 1$ represent the power to be applied in phase of starting up of the scooter with the model without $\mathrm{R}$ and $\mathrm{C}$.

\subsection{Simulation of the Proposed Model (with elements $\mathbf{R}$ and $\mathbf{C )}$}

The model bond-graph inverse with $\mathrm{R}$ and $\mathrm{C}$ is given by the following figure (fig.16):

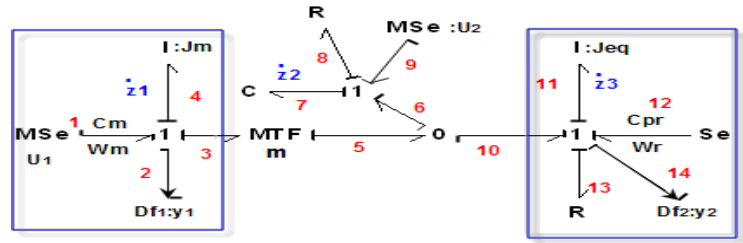

Fig.16.The inverse Bond-graph of proposed model (with R and $\mathrm{C}$ ).

By substituting the algebro-differential equations in the equations of the constraints, the controllers necessary to insure the equalization of the input and the output speeds, are given by the equation (3):

$$
\left\{\begin{array}{l}
U_{1}=p J_{m} y_{1}+\left(m * J_{e q} * p+R\right) y_{2} \\
U_{2}=m\left(R_{1}+\frac{c}{p}\right)\left(m y_{1}-y_{2}\right)-\left(J e q^{*} p^{*} y_{2}+R-C_{p r}\right) y_{2}
\end{array}\right.
$$

We impose on the model V1 the same conditions on the outputs (y1 and $\mathrm{y} 2)$ as the model $\mathrm{V} 0$ and we simulate the controls U1 and U2 via the inverse model (Fig.17 and Fig.18).

According to the equation (2) and by using SIMULINK we obtain the following simulations: 


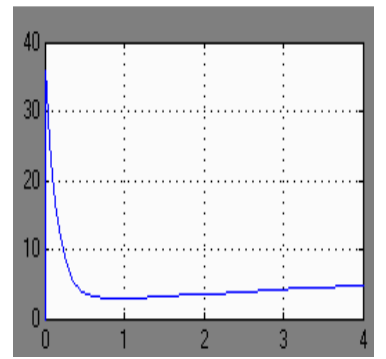

Fig.17.the U2 controllers for the model V1 (torque in N.m).

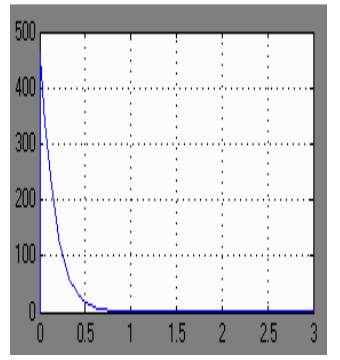

Fig.18.the U1 controllers for the model V1 (power in $\mathrm{W})$.

\subsection{Validation}

The control U2, which represents the torque to be applied to the Back wheel of scooter, is lower with the control U2 found in the model V0 for the same outputs y1 and y2.

By comparing models $\mathrm{V} 1$ and V0, we notice that $\mathrm{U} 1$ of the model V1 decreases with regard to that of V0, thus there are gains in term of power for the model V1 what shows and confirm the interest of our approach which is based on the addition of elements $\mathrm{R}$ and $\mathrm{C}$, from which their values are obtained by the optimization of the Gramians of controllability and of observability by using the genetic algorithm on energy criteria.

Indeed we deduce that input power of initial model is more important than proposed model one. Our approach offers the opportunity to explore more candidate solutions by adding bond graph elements.

The dimensioning of these elements in based on Gramians of controllability and of observability optimization. It allows the improvement of the performances with a systematic method.

\section{CONCLUSIONS}

In this paper we proposed a dimensioning procedure of mechatronic systems. This procedure uses collaborative tools such as bond graph and genetic algorithms. We integrated our contribution in a design methodology supported by functional structural and behavioral steps. We used energetic and control criteria which favorite interactions between passive and active parts. These criteria decrease confrontation between mechanical and control engineers in the preliminary design. This procedure was validated on our application automatic transmission of scooter. In this frame we demonstrated that the proposed model is more performed than the initial model. Thus we also can explore more candidate solutions and integrate this procedure in professional software for mechatronic design systems.

\section{REFERENCES}

[1] Pahl,W.Beitz ,(1996) "engineering design", Edited by K.Wallance, seconde edition , London:Springer , New York ISBN 03-540-19917-9.

[2] Robert H.Bishop "The mechatronic Handbook second Edition «Mechatronic systems control, logic and data acquisition" university of Texas at Austin

[3] Robert. H. Bishop (2003), the Mechatronics Handbook, , "Mechatronic Design Approach". University of Texas at Austin.

[4] Rosenberg, "Automated design approaches for multidomain dynamic systems using bond-graphs and genetic programming", In The International Journal of Computers, Systems and Signals (IJCSS), vol. 3, no. 1, 2002.

[5] Tollenaere,(1998) ,"conceptions des produits mécaniques-methodes, modeles et outils",Editions Hermes $N^{\circ}$ ISBN 2-86601-694-7.

[6] Wenhui Di, Bo Sun, Lixin Xu (2009) "Dynamic Simulations of Nonlinear Multi-Domain Systems Based on Genetic Programming and Bond Graphs" Tsinghua Science \& Technology, Volume 14, Issue 5, Pages 612616.

[7] Faiçal Miled, "Contribution à une méthodologie de conception des systèmes dynamiques actifs", Thèse université de technologie de Belfort-Monbliard.

[8] Rahmani, "Etude structurelle des systèmes linéaires par l'approche bon-graph", Thèse de doctorat de l'Université des Sciences et Technologies de Lile, 1993.

[9] Marx, D. Koenig et D. Georges, "Optimal Sensor and Actuator Location for Descriptor Systems using Generalized Gramian and Balanced Realization”, Proc. of the American Control Conference, 2004.

[10] Georges, D. (1995) "The use of observability and controllability Gramians or functions for optimal sensor and actuator location in finite-dimensional systems", Proceedings of IEEE Conference on Decision and Control, vol. 4 (pp. 3319\}3324).

[11] Marc van de Wal, Bram de Jager "A review of methods for input/output selection "Philips CFT, Mechatronic Motion, P.O. Box 218, SAQ-2116, 5600 MD Eindhoven, Netherlands "Faculty of Mechanical Engineering, Eindhoven University of Technology, P.O. Box 513, 5600 MB Eindhoven, Netherlands Received 11 June 1998; revised 3 July 2000; received in final form 6 September 2000.

[12] R.F. Ngwompo, S. Scavarda, “ Dimensioning problems in system design using bicausal bond-graphs", I.N.S.A. de Lyon, Laboratoire d'Automatique Industrielle, F69621 Villeurbanne Cedex, France Received 13 January 1999; received in revised form 1 May 1999.

[13] Dauphin Tanguy, Geneviève, "Les bond-graphs" (série système automatisés), Lavoisier 2000. 
[14] Zhun Fan, Jiachuan Wang, Sofiane Achiche, Erik Goodman, Ronald Rosenberg Structured synthesis of MEMS using evolutionary approaches"Applied Soft Computing, Volume 8, Issue 1, January 2008, Pages 579-589

[15] Kisung Seo, Zhun Fan, Jianjun Hu, Erik D. Goodman, Ronald C. Rosenberg" Toward a unified and automated design methodology for multi-domain dynamic systems using bond-graphs and genetic programming"Mechatronics, Volume 13, Issues 8-9, October 2003, Pages 851-885.

[16] F.S. Hover "Gradient dynamic optimization with Legendre chaos Automatic ", Volume 44, Issue 1, January 2008,Pages 135-140

[17] Goldberg, D.E., 1994, "Genetic Algorithms in Search, Optimization, and Machine Learning", Addison-Wesley Publishing, Reading, MA.

\section{BIOGRAPHIES:}

Amara Elhoucine, PHD Student in National School of engineering of Monastir, Monastir ,Tunisia, Houcine.Amara@enim.rnu.tn

Faiçal Miled, National School of engineering of Monastir Monastir,Tunisia,f.miled@uha.fr

Kamel BenOthman, National School of engineering of Monastir, Monastir, Tunisia, Kamelbenothman@yahoo.fr 